\title{
Review
}

\section{Small ubiquitin-like modifier protein-specific protease 1 and prostate cancer}

\author{
Yong Zuo, Jin-Ke Cheng \\ Laboratory for Cell Signal Transduction, Institute of Medical Sciences, Shanghai Jiao Tong University School of \\ Medicine, Shanghai 200025, China
}

\begin{abstract}
Small ubiquitin-like modifier protein (SUMO) modification is a highly dynamic process, catalyzed by SUMOspecific activating (E1), conjugating (E2) and ligating (E3) enzymes, and reversed by a family of SUMO-specific proteases (SENPs). There are six members of the human SENP family, and each SENP has different cellular locations and substrate specificities. However, the precise roles of SENPs in cellular processes have not been elucidated to date. This brief review will focus on recent advances pertaining to the identified targets of SENP1 and its potential role in prostate cancer.
\end{abstract}

Asian Journal of Andrology (2009) 11: 36-38. doi: 10.1038/aja.2008.45; published online 22 December 2008.

Keywords: SUMO, SUMO-specific protease, prostate cancer, androgen receptor, HIF1 $\alpha$

\section{SUMO and SUMOylation}

Small ubiquitin-like modifier (SUMO) was identified as a post-translational protein modifier over 10 years ago. Since then, hundreds of proteins have been reported as targets modified by SUMO, and the majority of these targets are nuclear proteins. SUMOylation can alter protein localization, activity or stability [1-3]. There are four members in the SUMO family, of which SUMO1-SUMO3 are ubiquitously expressed, whereas SUMO4 is mainly expressed in the lymph system. SUMO2 and SUMO3 are $97 \%$ identical, but share only $50 \%$ sequence identity with SUMO1 [1-3].

There are many similarities between ubiquitination and SUMOylation. However, the activating (E1), conjugating (E2) and ligating (E3) enzymes involved in SUMOylation

Correspondence to: Dr Jin-Ke Cheng, Laboratory for Cell Signal Transduction, Institute of Medical Sciences, Shanghai Jiao Tong University School of Medicine, Shanghai 200025, China.

Fax: +86-21-6466-1525 E-mail: jkcheng@shsmu.edu.cn

Received: 9 November 2008 Accepted: 10 November 2008

Published online: 22 December 2008 are entirely distinct from ubiquitin E1, E2 and E3 [3], and there is only one E1 for SUMOylation. E1 for SUMO is composed of two subunits, Aos1 and Uba2. In contrast to the large number of E2s used in the ubiquitination pathway, Ubc9 is the only SUMO-conjugating enzyme. E3 ligase can facilitate SUMO conjugation to catalyze the transfer of SUMO from UBc9 to a substrate. Several SUMO E3 ligases have been identified, most of which are characterized by a Siz/PIAS (SP)-ring motif, which is essential for their function. The ligases with an SPring motif can be subdivided into different groups, including the PIAS (protein inhibitor of the activator of signal transducers and activators of transcription protein [STAT]) family of proteins: MMS21, which is part of an octameric SMC5-SMC6 complex, and Zip3, which is the meiosis-specific yeast protein. Another type of E3 ligase is RanBP2, which is localized to the nuclear pore complex. The third class is Pc2, which is a component of the polycomb protein complexes $[1,2]$.

\section{De-SUMOylation}

SUMOylation can be reversed by a family of SUMO- 
specific proteases (SENPs) [2-5]. Two yeast SENPs, Ulp1 and Ulp2/Smt4, were characterized first. Both the enzymes not only deconjugate SUMO/Smt3 from modified proteins but also process $\mathrm{SUMO} / \mathrm{Smt} 3$ precursors to a mature form with a C-terminal diglycine. Sequence comparisons predicted an $\sim 200$-amino-acid protease fold, which defines this group of enzymes (C-48 cysteine proteases) [4, 5]. As such, there are six SENPs defined in mammalian cells [3]. The six members of the SENP family differ in their sequence homology and cellular location, as well as their substrate specificity, and can be divided into three subfamilies. SENP1 and SENP2 belong to the first sub-family and have broad substrate specificity. SENP1 is a nuclear protease that deconjugates a large number of SUMOylated proteins when overexpressed. The mutation of SENP1 causes embryonic lethality due to a defective hypoxiaHIF $1 \alpha$ pathway, which plays a crucial role in embryonic erythropoiesis [6]. SENP2 is a nuclear envelope-associated protease that, when overexpressed, appears to have an activity similar to that of SENP1. The second subfamily consists of SENP3 and SENP5, both of which are nucleolar proteins with preferences for SUMO2-SUMO3. SENP3 and SENP5 are reportedly involved in the regulation of cell mitosis. The third subfamily consists of SENP6 and SENP7, which have an extra loop in their catalytic domain [3-5]. Although SENPs have shown activity in the SUMOylation process, specifically in deconjugation, their precise physiological role in reversing SUMOylation has not been well defined.

\section{$3 \quad$ SENP1 and AR signaling}

$\mathrm{AR}$ is a ligand-regulated transcription factor belonging to the nuclear receptor superfamily. It mediates the effects of androgens on the regulation of cell growth, differentiation and maintenance of male reproductive functions. In the absence of ligands, AR is located primarily in the cytoplasm and associates with heat-shock proteins in an inactive state. On binding to the ligand, AR undergoes a series of changes, including conformational changes, translocation from the cytoplasm to the nucleus and binding to a specific DNA sequence called the androgen response element (ARE) in the promoter regions of target genes to regulate transcription of these genes [7,8]. AR also plays an important role in prostate cancer development.

Interestingly, AR and four of its co-regulators, SRC-1, SRC-2, p300 and histone deacetylase 1 (HDAC1), are conjugated by SUMO [9]. This feature makes AR signaling an attractive model to study the regulatory function of deSUMOylation. We initially performed a functional screening to determine which member of the SENP family regulates the cellular processes targeted by SUMOylated targets. Of these members, only SENP1 specifically and dramatically enhanced the AR-transcriptional activity. SENP1's ability to enhance AR-dependent transcription is mediated through deSUMOylation of HDAC1, thereby reducing its deacetylase activity and repressive activity to AR-dependent transcription [9, 10]. More interestingly, androgen is a regulator of SENP1 activity in this process [11]. We observed that exposure of LNCaP cells to androgen enhances SENP1 transcription. This androgenmediated augmentation of SENP1 is an AR-dependent event, as reflected by the absence of the effect in the presence of the androgen receptor antagonist bicalutamide, and in AR-negative prostate cancer PC-3 cells. We further identified the specific ARE on the promoter of the SENP1 gene that is required for SENP1 induction by androgens [11]. Therefore, SENP1 regulates androgen-AR signaling through a positive feedback mechanism.

\section{SENP1 and prostate cancer}

AR is one of the most important factors in prostate cancer development [12]. Enhanced AR activity is essential for cancer cell growth because prostate cancer, in most cases, will regress in response to androgen removal therapy [12]. We have determined that SENP1 enhances androgen-induced AR activity, and also that SENP1 expression can be conversely induced by androgen-AR signaling, which further enhances AR activity through a positive feedback mechanism. Such a relationship between SENP1 and AR signaling prompted us to explore a potential role of SENP1 in prostate cancer development. In particular, we examined the expression of SENP1 in prostate cancer specimens. We found that SENP1 was over-expressed in over $50 \%$ of more than 100 specimens with high-grade prostatic intraepithelial neoplasia (PIN) and prostate cancer [10]. In addition, the role of SENP1 overexpression in the development of prostate cancer has been confirmed in SENP1 transgenic mice. We generated probasin-driven murine SENP1 transgenic mice and showed increased proliferation of prostate epithelial cells at 3 months of age. Importantly, a significant increase in PIN-like structure formation was observed in SENP1 transgenic mice that were older than 4 months [10]. These results strongly support a role of SENP1 in prostate tumorigenesis.

\section{SENP1 and hypoxia-HIF1 $\alpha$ signaling}

We generated SENP1 knockout mice to delineate the contribution of SENP1 in development [6]. SENP1 ${ }^{-1-}$ mice were shown to exhibit embryonic lethality between E13 and E15. We observed a marked decrease in the number of erythropoietic foci in the fetal liver of SENP $1^{-/-}$mice, indicating that the activity of definitive erythropoiesis was greatly decreased. Furthermore, the deficiency in erythropoiesis was confirmed to occur in committed erythro- 
poietic precursors beyond the erythroid colony-forming unit (CFU-E) stage (Ter- $119^{+}$cell), which was significantly decreased in SENP1 ${ }^{-1-}$ mice due to apoptosis [6]. We observed that the expression of Epo, a crucial factor for erythroid or CFU-E survival, was decreased in SENP1 ${ }^{-/}$fetal liver. We further determined the contribution of Epo in SENP1 $1^{--}$mice by adding Epo, which efficiently increased survival and decreased apoptosis of SENP1 $1^{-1}$ erythroid [6].

HIF1 $\alpha$ is a member of the bHLH-PAS (basic helix loop helix-Per-Arnt/AhR-Sim) family of proteins and binds to canonical DNA sequences (hypoxia-regulated elements) in the promoter or enhancer regions of the target genes [13]. Epo is one of the HIF1 $\alpha$ target genes. We have confirmed that SENP1 regulates Epo expression in an HIF1 $\alpha$-dependent manner. SENP1 can de-conjugate SUMOylated HIF1 $\alpha$ induced by hypoxia. Interestingly, the de-SUMOylation activity of SENP1 is essential for SENP1-mediated regulation of Epo transcription, indicating that SENP1 regulates Epo expression through de-conjugation of SUMOylated HIF1 $\alpha$ [6]. Hypoxia is the established inducer of HIF $1 \alpha$ stabilization and Epo expression [13-16]. The stability of the HIF1a response to hypoxia is significantly decreased in SENP1 ${ }^{-/}$ mice [6]. These results show an important role of SENP1 in maintaining hypoxic-induced stabilization of HIF $1 \alpha$ protein.

The significance of these findings can be extended to the potential role of SENP1 in tumor angiogenesis because tumor cell expansion relies on nutrient supply. Oxygen limitation is therefore central in controlling angiogenesis, glucose metabolism, tumor survival and tumor spread. This pleiotropic action is orchestrated by $\mathrm{HIF} 1 \alpha$, which is a master transcriptional factor in hypoxia signaling. The expression of vascular endothelial growth factor (VEGF), an angiogenic factor in the hypoxia-HIF $1 \alpha$ process, in response to hypoxia, is significantly decreased in SENP $1^{-1}$ MEF cells [6], indicating that SENP1 is involved in the regulation of hypoxia-induced expression of VEGF. We are currently investigating the role of SENP1 in this mechanism by analysis of tumor samples and animal models.

There are over 200 SUMOylated target proteins reported [1]. Given this, we have used different strategies to determine some of the targets of SENP1 and the cellular processes involved. As these targets are related to tumorigenesis, we speculate that SENP1 plays an important role in cancer development. However, we are still uncertain as to the clinical significance of SENP1 and its involvement in tumorigenesis. We are currently using tumor specimens to analyze the relationship among SENP1, its targets and clinical features of the tumor. It is likely that in the years ahead, we will confirm the contributions of SENP1 to prostate cancer tumorigenesis.

\section{Acknowledgment}

Studies in the author's laboratory were funded by startup funds from the Shanghai Jiao Tong University School of Medicine, National Natural Science Foundation of China (No. 30772462). Most of the work described in this review was performed in Dr Edward Yeh's laboratory at the MD Anderson Cancer Center, Houston, TX, USA.

\section{References}

1 Geiss-Friedlander R, Melchior F. Concepts in sumoylation: a decade on. Nat Rev Mol Cell Biol 2007; 8: 947-56.

2 Hay RT. SUMO: a history of modification. Mol Cell 2005; 18 : $1-12$.

3 Yeh ET, Gong L, Kamitani T. Ubiquitin-like proteins: new wines in new bottles. Gene 2000; 248: 1-14.

4 Hay RT. SUMO-specific proteases: a twist in the tail. Trends Cell Biol 2007; 17: 370-6.

5 Mukhopadhyay D, Dasso M. Modification in reverse: the SUMO proteases. Trends Biochem Sci 2007; 32: 286-95.

6 Cheng J, Kang X, Zhang S, Yeh ET. SUMO-specific protease 1 is essential for stabilization of HIF1alpha during hypoxia. Cell 2007; 131: 584-95.

7 Gelmann EP. Molecular biology of the androgen receptor. J Clin Oncol 2002; 20: 3001-15.

8 Shang Y, Myers M, Brown M. Formation of the androgen receptor transcription complex. Mol Cell 2002; 9: 601-10.

9 Cheng J, Wang D, Wang Z, Yeh ET. SENP1 enhances androgen receptor-dependent transcription through desumoylation of histone deacetylase 1. Mol Cell Biol 2004; 24: 6021-8.

10 Cheng J, Bawa T, Lee P, Gong L, Yeh ET, et al. Role of desumoylation in the development of prostate cancer. Neoplasia 2006; 8: 667-76.

11 Bawa-Khalfe T, Cheng J, Wang Z, Yeh ET. Induction of the SUMO-specific protease 1 transcription by the androgen receptor in prostate cancer cells. J Biol Chem 2007; 282: 37341-9.

12 Debes JD, Tindall DJ. The role of androgens and the androgen receptor in prostate cancer. Cancer Lett 2002; 187: 1-7.

13 Wang GL, Jiang BH, Rue EA, Semenza GL. Hypoxia-inducible factor 1 is a basic-helix-loop-helix-PAS heterodimer regulated by cellular O2 tension. Proc Natl Acad Sci USA 1995; 92 : 5510-4.

14 Huang LE, Gu J, Schau M, Bunn HF. Regulation of hypoxiainducible factor 1 alpha is mediated by an $\mathrm{O}_{2}$-dependent degradation domain via the ubiquitin-proteasome pathway. Proc Natl Acad Sci USA 1998; 95: 7987-92.

15 Ivan M, Kondo K, Yang H, Kim W, Valiando J, et al. HIF alpha targeted for VHL-mediated destruction by proline hydroxylation: implications for O2 sensing. Science 2001; 292: 464-8.

16 Maxwell PH, Wiesener MS, Chang GW, Clifford SC, Vaux $\mathrm{EC}$, et al. The tumour suppressor protein VHL targets hypoxiainducible factors for oxygen-dependent proteolysis. Nature 1999; 399: 271-5. 